\title{
Corela
}

Cognition, représentation, langage

HS-12 | 2012

Langue, espace, cognition

\section{Locus et telos : aller à l'école, être à la plage}

\section{Francis Corblin}

\section{OpenEdition}

\section{Journals}

Édition électronique

URL : http://journals.openedition.org/corela/2722

DOI : $10.4000 /$ corela.2722

ISSN : 1638-573X

\section{Éditeur}

Cercle linguistique du Centre et de l'Ouest - CerLICO

\section{Référence électronique}

Francis Corblin, «Locus et telos : aller à l'école, être à la plage », Corela [En ligne], HS-12 | 2012, mis en ligne le 19 décembre 2012, consulté le 01 mai 2019. URL : http://journals.openedition.org/corela/2722 ; DOI : 10.4000/corela.2722

Ce document a été généré automatiquement le 1 mai 2019.

\section{(c) (i) (2)(2)}

Corela - cognition, représentation, langage est mis à disposition selon les termes de la licence Creative Commons Attribution - Pas d'Utilisation Commerciale - Partage dans les Mêmes Conditions 4.0 International. 


\title{
Locus et telos: aller à l'école, être à la plage
}

\author{
Francis Corblin
}

1 Quelques expressions de localisation, parmi les plus courantes du français, présentent des propriétés remarquables. A la question « Où va (est) Pierre ? », on peut répondre :

(1) Pierre va à l'école.

(2) Pierre est à la plage.

2 Les propriétés particulières de ces phrases sont les suivantes

a. Il ne s'agit pas seulement de localisation. Chacune de ces phrases évoque en outre, et surtout en l'absence de tout autre information sur l'individu désigné, la participation de Pierre à une "routine sociale » (Vandeloise 1987) on comprendra, le plus souvent en (1) que Pierre est un écolier, et en (2) que Pierre profite des plaisirs de la plage.

b. l'analyse du défini complément n'est pas simple s'agit-il d'un générique, d'un emploi « intensionnel » (Furukawa, 1986-2010)?

c. la nature compositionnelle de ces constructions est douteuse. Beaucoup, sans doute, auraient envie d'y voir des idiomes, ou évoqueraient un type particulier $d^{\prime}$ '« incorporation » du défini complément au verbe.

3 Les phrases de localisation qui manifestent ces propriétés en français utilisent des verbes de localisation peu spécifiés (être, aller), avec un sujet humain, un complément défini singulier, et des noms typiquement associés à des routines sociales (hôpital, plage).

4 Cet article propose une analyse de ces constructions qui permette d'expliquer qu'elles manifestent, typiquement, une dualité d'interprétations une interprétation de localisation spatiale (locus, Pierre est localisé dans un lieu, ou se rend dans un lieu) et une interprétation télique (telos, Pierre va ou se rend dans ce lieu, pour y bénéficier de la fonction sociale associée). 


\section{La nature du défini complément}

5 Deux courants de recherche ont noté le statut particulier de ce défini. Il est analysé comme " générique »; dans la plupart des travaux sur les prépositions de lieu (Vandeloise 1987, Aurnague 2004, entre autres). Furukawa (dès son livre de 1986, à la suite de Martin 1986) parle de défini «intensionnel » et en donne une analyse soucieuse de noter ses similarités, mais aussi ses différences avec le défini générique.

6 Ces deux courants de recherche partagent l'idée que ce défini n'est pas « référentiel ", qu'il n'est pas utilisé pour dénoter un objet ou lieu particulier. Le problème, pour cette vue classique, est qu'elle ne permet en aucune manière de prédire que ce GN défini peut être utilisé, tout en gardant son interprétation " routine sociale », comme antécédent de reprises anaphoriques ou démonstratives, ou comme antécédent de lequel:

(3) Pierre va (est) à l'école.

a. Et cette école est loin de votre domicile?

b. Et à laquelle l'avez-vous inscrit?

7 La plupart des théories classiques de l'anaphore (voir par exemple Corblin 2002) admettent que ces suites ne sont acceptables que si un groupe nominal de la phrase antérieure a introduit un individu particulier dans le discours. Aucune théorie, à ma connaissance, n'admet que l'antécédent de ces expressions puisse être généré sur la base d'inférences pragmatiques.

8 Le seul groupe nominal de la phrase qui peut être à la source de cette introduction de particulier est évidemment le groupe nominal défini singulier. Il en résulte comme conséquence nécessaire que ce groupe nominal ne peut être ni un générique, ni un groupe nominal incorporé, ni un groupe nominal partie d'une expression idiomatique.

9 Schématiquement, on peut opposer les deux séries suivantes, qui manifestent les différences les plus significatives entre la construction qui nous occupe, être à l'hôpital, et une expression qui est souvent donnée comme « exprimant » l'interprétation télique de cette construction, être hospitalisé :

(4) a. Marie est à l'hôpital

Et elle y reste longtemps.

Et où est cet hôpital?

Et dans lequel?

(4) b. Marie est hospitalisée

? Et elle y reste longtemps?

? Et où est cet hôpital ?

* Et dans lequel?

Il faut admettre que (4)a, même quand il implique (4)b, introduit, ce que ne fait pas (4)b, un particulier dans le discours par l'intermédiaire du GN défini. Aucune analyse reposant sur la généricité, ou l'intensionalité du défini dans ces tournures, qui expriment, c'est vrai, un peu plus que la simple localisation, ne peut prédire ces faits.

11 Quelle analyse pourrait-on alors proposer pour le défini de ces constructions? Il s'agit d'un défini qui introduit un particulier, mais peut s'utiliser sans que les locuteurs puissent identifier le particulier, et sans d'ailleurs qu'ils s'intéressent vraiment à son identité.

12 Un emploi du défini a ces propriétés, le défini dit « associatif », ou « fonctionnel », illustré en (6): 
(6) Marie tomba malade, et le médecin lui prescrivit du repos. décrite pour savoir qu'elle est de celles où, typiquement, la fonction associée au nom est définie (s'applique à un individu). Dans un exemple comme (7), c'est l'usage de blessé qui légitime l'usage du défini fonctionnel l'hôpital :

(7) On achemina le blessé vers l'hôpital.

Mais ce n'est pas si strict; il y a des emplois sans indice préalable; il suffit parfois que le prédicat de la phrase soit compatible avec l'activité typique associée au nom pour que le défini associatif soit légitimé, comme en (8) :

(8) Le médecin m'a dit d'arrêter de fumer.

Comparons avec (9) :

(9) Un médecin m'a dit d'arrêter de fumer.

Le défini en (8) est légitimé par le fait qu'un tel conseil suffit à identifier un médecin et un seul. Cela est réalisé si ce médecin est celui que j'ai consulté pour ma santé et non, par exemple, un médecin rencontré au café, ce que (9) autorise, mais non (8).

Médecin est lexicalement défini comme un rôle ou fonction soigner les gens. Le médecin, même en l'absence d'indices contextuels impliquant blessure ou maladie, et si le prédicat ne s'y oppose pas, sera interprété comme l'individu tenant du rôle, et consulté par l'actant humain mentionné dans la phrase pour sa santé.

19 Convenons de parler de défini " auto-légitimé » pour les emplois illustrés en (8). On y utilise un défini singulier fonctionnel en l'absence de tout indice externe indiquant que la situation décrite dans la phrase est de celles qui typiquement rendent le terme fonctionnel utilisable pour un individu. C'est le contenu de la phrase elle même, qui légitime son usage. Il faut pour cela que la fonction associée au nom-tête du défini trouve dans la phrase un prédicat normalement associé à la fonction. Dans un exemple comme (10), l'auto-légitimation ne fonctionne pas parce que le prédicat n'a aucun rapport avec la fonction typique des médecins. La conséquence est que ce défini a besoin d'une légitimation externe, soit par association à un élément du contexte, soit par reprise :

(10) Le médecin cria pour effrayer le corbeau.

Ce rapprochement entre les tours locatifs placés au centre du travail (être à l'hôpital, aller à la plage), et l'usage auto-légitimé de certains définis fonctionnels (le médecin m'a prescrit du repos) nous amène à l'hypothèse suivante : dans les tours locatifs en débat, le recours au défini implique que la seule localisation à une entité de type $\mathrm{N}$ (hôpital, plage) suffit, en l'absence d'autre information, à identifier un individu $\mathrm{N}$ et un seul celui qui remplit, à l'égard de l'humain mentionné dans la phrase, sa fonction typique. Nous tenterons dans ce qui suit de donner corps à cette hypothèse. 


\section{Un essai de dérivation informelle de l'interprétation}

21 Toutes les phrases du type considéré peuvent simplement localiser dans l'espace l'entité sujet par rapport à un site particulier, mentionné dans le complément :

(11) Le taxi est à la plage.

(12) Pierre est à la plage.

Pierre, au même titre que le taxi, est simplement localisé dans un lieu particulier. Il s'agit de la partie locus de l'interprétation, celle qui émerge de manière tout à fait générale, quelle que soit la cible (humain ou non), si le site peut être interprété comme un nom de lieu (et non d'objet, cf. infra), et qui a été étudiée par Vandeloise (1987), et Aurnague (2001, 2004, 2009, à paraître).

Mais Pierre est un actant humain, et plage serait sans doute aujourd'hui défini dans le lexique, comme endroit au bord de l'eau dans lequel les humains se rendent pour profiter d'activités de loisir et de détente propres à ce lieu. Ici apparaît le telos.

L'idée est la suivante: en l'absence d'indications externes, l'emploi du défini présume qu'il est auto-légitimé. Cela implique que la situation décrite par la phrase est de celles qui justifient l'emploi du défini fonctionnel: le particulier en cause est identifiable comme l'institution-lieu de type "plage » dans laquelle Pierre se rend pour y bénéficier des plaisirs associés.

Cela explique que (12) dise beaucoup plus, en général, que la simple localisation (11), et que l'identité particulière de la plage en question passe au second plan exactement comme l'identité du médecin particulier en (8).

Dans les usages de pure localisation, comme en (11), le défini n'est légitimé que si des facteurs externes, connaissances partagées ou texte antérieur, permettent de présumer que l'interlocuteur est en mesure d'identifier préalablement à l'usage de cette phrase, et indépendamment de ce qu'elle dit, un individu particulier de type " plage ».

Ce qui est remarquable en (12) est que deux choses se produisent en même temps. Tout d'abord, le défini semble légitimé par l'énonciation de la phrase cela signifie qu'aucune connaissance externe n'est requise pour que les locuteurs acceptent la phrase ; comme parfaitement naturelle. En deuxième lieu, l'interprétation n'est plus dans ce cas de pure localisation dans un lieu du type considéré nous comprenons en outre que l'actant animé est dans un lieu de ce type pour s'y livrer à l'activité typiquement associée à ce lieu.

Considérons un bref exemple pour illustrer. Supposons que nous habitions un petit village. Il y a une seule école que nous connaissons. Une phrase comme (13) peut être purement locative :

(13) Pierre est à l'école.

Pierre est peut-être parti faire du vélo devant l'école, il est peut-être l'ami de l'institutrice qui habite là, etc...

30 Mais (13) peut aussi avoir une interprétation télique : le locuteur est conduit à inférer que Pierre est dans une école (celle que nous connaissons ou une autre) en tant qu'écolier.

31 Je rejoins ici Borillo (2001) et Aurnague (2001) qui soulignent que dans certains emplois, les deux usages (locatif et routine sociale) peuvent apparemment se superposer. C'est le cas par exemple si j'utilise (13) pour dire que Pierre est à l'école (que nous connaissons) et qu'il y est en classe. 
L'usage locatif peut donc exister sans être accompagné nécessairement par l'interprétation routine sociale, bien que cette dernière tende à s'imposer pour les actants humains pour lesquels elle est plausible.

Si l'interprétation de routine sociale s'impose, tout se passe comme si la définitude du GN défini changeait de nature il ne s'agit plus d'un individu école identifié par des critères externes, mais d'un individu école identifié en tant que tenant d'un rôle à l'égard de l'actant humain. Ainsi, dans le même contexte que (13), je peux parfaitement parler de Pierre qui est à l'école à l'autre bout du monde, bien que l'école soit en mesure d'identifier une seule école (l'école unique de notre village).

Si l'interprétation télique est retenue, l'individu est F-identifié en tant qu'institution sociale, et la localisation spatiale proprement dite devient indéfinie l'interprétation télique de (13) est correcte même si le locuteur ne suppose pas que l'interlocuteur sait dans quelle école est Pierre. On représenterait assez fidèlement l'interprétation télique de (13) comme la conjonction de a et b

- a. Pierre est à l'école-institution pertinente pour son statut d'élève.

- b. Pierre est dans une école.

Certains proposeraient même que la composante b soit traitée comme une implicature, non une partie du sens, au vu d'exemples comme :

(14) Pierre est à l'école, mais pas dans une école. Il travaille en alternance.

Tous les noms en question sont des noms qui s'appliquent à des activités sociales associées à des lieux dédiés. Une entrée de dictionnaire plausible pour école pourrait être (15) :

(15) Ecole

1. Institution sociale destinée à enseigner des savoirs aux enfants \& disposant (en général) de lieux réservés à cet usage.

2. Lieu dédié au fonctionnement de l'institution sociale « école ».

Cela détermine deux facettes pour les noms de ce type, illustrées dans les exemples suivants :

Ma femme est restée à la maternité : insitution

Ma voiture est restée à la maternité deux jours. : lieu

Une définition comme (15) permet d'intégrer tous les cas mentionnés. Les exemples purement locatifs, comme le taxi est à l'école, doivent naturellement sélectionner la facette lieu. Les cas d'interprétation télique comme Pierre est à l'école sélectionnent l'interprétation institution sociale, et héritent alors en vertu de l'entrée lexicale (15) de la lecture par défaut que cela implique, en général une localisation dans un lieu dédié. Mais s'agissant d'une inférence par défaut, des usages tels que (14) sont légitimés.

Résumons cette approche à grands traits.

Un certain nombre d'unités lexicales s'appliquent à des institutions sociales qui s'exercent dans des lieux réservés (école, hôpital), ou à des lieux voués socialement à un certain type d'activité (plage). Ces unités lexicales polysémiques, selon la facette qui est sélectionnée par le contexte, auront deux interprétations possibles: elles peuvent évoquer un lieu ayant une identification spatiale et une seule, et elles acceptent alors tous les prédicats applicables à ces entités (occuper x m2, être repeint, ratissé, etc...), ou une institution-activité qui n'est pas définie par le lieu où elle s'exerce, mais par une fonction, bien que ces activités aient des lieux réservés, acceptant alors d'autres prédicats (être transféré, changer de superficie, etc.). 

témoigne le contraste entre (17) et (18) :

(17) L'école du village a été repeinte en blanc.

(18) L'école du village donne aux enfants une excellente formation. « école » ou « hôpital » pour cet actant). les conditions pour qu'il en soit ainsi (il est blessé ou malade).

\section{Problèmes et questions} il s'agit d'une pure localisation :

(16) Pierre est dans l'école. conçu comme un lieu d'un certain type. sociale ", qui est l'élément crucial pour les emplois fonctionnels.

Avec des constructions de localisation (être à, aller à) le complément défini singulier peut s'interpréter comme renvoi à la facette «lieu», et doit alors être légitimé par une possibilité d'identification de l'individu considéré. Ce complément défini singulier, avec des actants humains, s'interprète comme l'individu particulier remplissant le rôle de l'institution sociale pertinent pour l'individu en question (l'entité qui remplit le rôle

Dans ce dernier usage, le défini est de type fonctionnel (associatif). La particularité de ces constructions locatives est que ce défini fonctionnel est auto-légitimé, et n'a aucun besoin d'informations antérieures nous indiquant que l'actant humain se trouve dans une situation telle qu'elle rend saillant le rôle pertinent. En d'autres termes, la seule différence que nous postulons entre «Pierre était blessé. Il alla à l'hôpital » et « Pierre est à l'hôpital » est que dans le second cas, c'est la seule localisation de Pierre par rapport à une entité de ce type présumée identifiable qui nous amène à inférer que Pierre remplit

\subsection{Pourquoi l'interprétation télique disparaît-elle avec les autres prépositions de lieu?}

Il suffit de remplacer à par une autre préposition de lieu pour que l'interprétation télique ne soit plus accessible. Dans la phrase (16), il n'est pas impliqué que Pierre soit écolier, et

43 L'hypothèse que l'on peut faire est que les autres prépositions de lieu sont plus spécifiées et spécialisées et imposent une forte restriction de sélection sur leur objet qui doit être

4 Les $\mathrm{N}$ discutés jusqu'à présent ont deux facettes (lieu/institution sociale), comme en

Une hypothèse plausible serait que les autres prépositions de lieu ne voient que la facette « lieu concret » dans ces unités lexicales et n'ont pas accès à la facette «institution

La généralisation induite est que l'interprétation télique n'est ouverte que par les prépositions qui ne sélectionnent pas l'interprétation de lieu concret pour leur complément et admettent une localisation plus «abstraite » de la cible, une localisation par relation d'implication dans une institution-activité.

4 L'idée est que à peut localiser par relation à un lieu (comme les autres prépositions locatives), mais peut aussi localiser par relation à une institution ou activité sociale, est confirmée par des exemples tels que :

(19) Pierre va (est) au travail.

(20) Pierre est au repos.

(21) Nous étions à l'étude quand le proviseur entra (...)

(22) Je suis (vais) à la gym. 

qu'il s'agit d'une préposition admettant dans ses contraintes de sélection de se combiner avec un $\mathrm{N}$ dénotant une activité. Cet usage est prohibé pour les pures prépositions de lieu, qui ne donnent donc pas lieu à interprétation télique.

\subsection{Activités-lieux et activités-objets}

M. Aurnague (à paraître) met en avant une série très intéressante d'exemples impliquant non plus des institutions-lieux, comme école, ou hôpital, mais des objets, comme piano, four:

(23) Marie est au piano.

(24) La dinde est au four

Il rappelle, en substance, qu'avec ces noms, il n'y a pas d'interprétation de localisation spatiale possible, comme le montre (25):

(25) * La mouche est au piano.

En revanche, si (23) et (24) sont corrects, c'est seulement avec une interprétation de routine sociale, ou d'activité, qui enrichit considérablement la localisation par rapport à un piano ou à un four ; nous comprenons que Marie est devant le piano pour en jouer, et que la dinde est à l'intérieur du four pour être cuite.

. Aurnague, je pense qu'il s'agit du même phénomène (version « objet») que celui que j'ai analysé pour des institutions-lieux comme école ou hôpital dans Corblin (2011), et je propose de le désigner sous le même terme d'interprétation « télique ».

Plusieurs points importants sont à considérer.

Que le phénomène ait lieu pour des noms avec lesquels il n'y a pas d'interprétation locative possible avec à, confirme que l'interprétation télique n'est pas crucialement liée à une localisation spatiale, dont elle ne serait que la sur-détermination les interprétations téliques semblent directement générées par le fait que à admet de localiser une cible par implication dans une activité. Il existe donc, comme le montrent les exemples considérés par M. Aurnague, des interprétations téliques avec à, pour des structures qui n'ont pas d'interprétation locative correspondante. lieux une véritable polysémie du nom-tête. Peut-on, par exemple, définir l'unité lexicale piano par en termes de "facettes » (objet concret ou activité consistant à jouer de cet objet) ? C'est un peu problématique dans la mesure où l'une des interprétations (activité) n'apparaît en général avec aucun autre déterminant que le défini un piano, deux pianos, tout piano ne quantifient que sur des objets concrets. Beaucoup de piano, un peu de piano, du piano, impliquent en revanche, et sans ambiguïté, une activité, mais il s'agit de termes massifs, et le caractère massif/comptable étant un trait de l'identité des noms on dirait plutôt qu'il s'agit de noms homonymes. C'est seulement «le piano », de manière générale, qui admet d'être interprété comme référence à une activité ou à un objet :

(26) Le piano est une passion dévorante.

(27) Le piano, c'est de $8 \mathrm{~h}$ à $10 \mathrm{~h}$ (dans un conservatoire). précédés de l'article défini, de s'interpréter comme référence à l'activité à laquelle est 
typiquement destiné l'objet en question. Cela n'est pas vrai pour tous les noms d'objets à interprétation télique, mais au moins pour nombre d'entre eux.

Pour résumer de manière générale, les noms d'objets impliqués dans ces interprétations téliques désignent des objets concrets (un piano, tout piano), non les activités auxquelles ils sont destinés ; cependant, avec l'article défini, on rencontre des usages qui réfèrent à des objets, et des usages qui réfèrent à l'activité associée (cf. 26-27).

Pouvons-nous, pour autant, en conclure que dans les interprétations téliques de être au piano, être au four, le piano et le four désignent des activités? Je pense que ce n'est pas le cas si la phrase dans son ensemble localise bien par rapport à une activité, le GN lui-même introduit un objet concret. Plusieurs arguments l'établissent. Tout d'abord, l'interprétation télique se maintient avec des déterminations concrètes de l'objet :

(28) Marie était au piano de Jean.

(29) La dinde était au four de la cuisine.

D'autre part, toute continuation anaphorique traite l'entité introduite comme une chose, non comme une activité :

(30) Marie était au piano. Ce piano lui venait de son oncle.

* Ce piano lui prit une heure.

(31) Marie était au piano.

Lequel? Celui de son oncle.

Il n'est pas, en outre, très facile de produire des exemples homologues de Pierre est à l'école , mais pas dans une école, c'est-à-dire des exemples compatibles avec le fait que la phrase n'implique pas relation à un objet concret du type mentionné.

Ce qui distingue les objets des lieux, c'est donc précisément que l'interprétation de pure localisation spatiale est impossible pour les objets avec la préposition à (A), et qu'il n'est pas très justifié de traiter les noms d'objet comme sièges d'une polysémie objet/activité associée (B)

- A. à locatif sélectionne un lieu et exclut les objets (Aurnague, à paraître)

- Le taxi est à l'école.

${ }^{*}$ La mouche est au fauteuil.

- B. Les noms d'institution-lieu possèdent plusieurs facettes. Il est difficile de soutenir que les noms d'« objets-activités » à interprétation télique le sont

- Une école peut s'installer en ce lieu

*Un piano peut prendre du temps

61 Pour ces noms il existe une version objet (comptable, un piano) et une version activité (massive, du piano), mais c'est la version « objet » qui est utilisée dans les interprétations téliques.

62 Je propose de traiter les interprétations téliques des objets comme un "forçage ", (l'interprétation de pure localisation étant exclue), qui superpose une interprétation " activité associée » à l'interprétation " objet». Le mécanisme se décompose ainsi. La préposition à admet deux constructions :

- (a) Une construction locative : son complément doit être un lieu, non un objet. (Le taxi est à la plage, ${ }^{*}$ La mouche est au fauteuil).

- (b) Une construction télique : son complément doit dénoter une institution sociale, ou une activité. (Marie est à la gym).

63 Avec les objets, la phrase sera néanmoins acceptable s'il est possible d'interpréter la phrase comme localisation de l'actant-sujet en tant que participant à l'activité 
typiquement associée à cet objet. Considérons l'exemple de four. L'interprétation locative est exclue La mouche est au four ne peut pas s'interpréter comme «la mouche est en proximité-contiguïté spatiale avec le four. »

L'interprétation télique consiste à considérer l'activité à laquelle on destine un four, et à interpréter l'actant-sujet comme participant impliqué par cette activité, c'est-à-dire quelque chose que l'on met à cuire. Elle sera donc plus plausible pour gigot que pour mouche pour des raisons purement pragmatiques tenant à ce que nous savons de l'alimentation des humains.

La thèse est donc que la préposition à, qui admet, en général, de prendre pour argument des activités (voir supra), et non des objets, combinée avec des arguments dénotant des objets, force, dans certains cas, leur interprétation comme activité.

Si le phénomène est bien à analyser ainsi, comme "forçage ", on devrait en trouver des confirmations indirectes. On s'attend, par exemple, à ce qu'il s'agisse d'un mécanisme qui s'applique de manière irrégulière, et non systématique, aux noms d'objet à activité associée, et c'est bien ce qu'on observe. Considérons par exemple des noms d'outils marteau, tournevis, pinceau. Les locuteurs que j'ai interrogés doutent de la possibilité d'utiliser ces noms avec $\grave{a}$ :

(32) ? Pierre est au marteau (tournevis, pinceau).

67 Même avec les noms d'instruments de musique, beaucoup font une différence entre être au piano, qui leur semble utilisable en n'importe quelle circonstance, et être à la guitare, être à la flûte à bec, qui leur semblent d'un usage plus restreint. Ce que disent les locuteurs est qu'avec la plupart des instruments de musique, l'usage de à suppose que le contexte mentionne une formation musicale dans laquelle l'instrument en question est une composante typique. Ce cas évoque naturellement l'usage de à avec les parties constitutives d'un tout :

(33) ? La mouche est au volant.

(34) Pierre est au volant.

68 Ce que suggèrent ces exemples, c'est que dans le cas d'entités fonctionnelles (orchestre, véhicule) définissant typiquement des sous-parties constitutives par des rôles dans l'activité ou le mécanisme, il sera aisé d'interpréter la désignation des objets constituants comme renvoyant aux activités auxquelles ils sont destinés.

69 Ce qui me semble pertinent, en l'occurrence, c'est que ces contextes favorisent fortement l'interprétation «fonction dans une activité ", par rapport à la simple désignation d'un objet. Il suffit parfois de mettre en place un scénario remplissant ces conditions pour que ce qui était difficilement acceptable devienne parfait :

(35) Pour mettre en place ces vis, il fallait d'abord les introduire d'un coup de marteau, puis les visser avec le tournevis. Ils se répartirent les tâches Pierre était au marteau, et Marie au tournevis.

70 J'ajoute que même pour les sous-parties reconnues d'un tout, l'association à une activité typique est cruciale :

(36) Pierre était au volant, ?son épouse au siège passager, ses enfants à la banquette arrière, et ses bagages au coffre.

71 C'est sans aucun doute parce que le volant est associé à une action, celle de conduire, donc à une activité, que l'on perçoit ces différences d'acceptabilité, car les termes volant, siège passager, banquette arrière ne se distinguent en rien en ce qui concerne leur capacité à légitimer des anaphores associatives ou fonctionnelles. 
72 En outre, on observe des irrégularités difficilement prévisibles qui sembleraient confirmer qu'il ne s'agit pas d'un mécanisme primitif de la grammaire, mais d'un forçage apparaissant assez irrégulièrement. Ainsi, si place du mort et siège passager désignent une seule et même entité dans une voiture, (37) est parfait, et (38) bizarre.

(37) Pierre était à la place du mort.

(38) ? Pierre était au siège passager.

De même, on comprend mal quelles raisons de principe expliqueraient des contrastes comme :

(39) Le beurre est au réfrigérateur. / ? L'huile est à l'armoire.

(40) Pierre est à la télé. / ? Marie est à la radio. ? Marie est au lecteur de DVD.

Si en revanche on traite ces interprétations, non comme des options ouvertes par la grammaire, mais comme des forçages qui imposent localement une interprétation "activité " à des noms d'objets, il est compréhensible que le phénomène soit linguistiquement aléatoire, et ne concerne pas tous les objets associables lexicalement à des activités.

Dans l'ensemble, le forçage télique est donc favorisé si l'objet est associé à une activité dans un fonctionnement plus large. Mais l'accès à cette interprétation pour les noms d'objet semble irrégulier et difficile à prédire avec certitude.

Il est sans doute utile de revenir sur le statut du défini dans les exemples impliquant des objets. Tout ce que nous venons d'observer indique que l'interprétation télique est favorisée dès que le contexte permet de traiter le défini comme défini fonctionnel (cf. le cas de l'orchestre ou du véhicule). C'est une double confirmation de nos analyses, car (1) cela confirme que le défini des interprétations téliques est exactement du même type que le défini associatif, et (2) cela confirme qu'à la différence des institutions-lieux, il est requis pour les objets que le contexte joue un rôle, en rendant saillante cette interprétation fonctionnelle, qui seule leur permet d'être interprétés comme activités.

\subsection{Le statut de la télicité}

\subsubsection{Lieux-institutions}

Le fait que ces tournures sont liées à l'interprétation télique du nom tête du défini est, à ma connaissance, une suggestion originale d'A. Borillo (2001), formulée dans le cadre du lexique génératif (Pustejovsky, 1995). Toutes mes observations la confirment l'interprétation non purement locative survient pour des $\mathrm{N}$ associés à des institutions sociales définies par leur fonction dans l'intérêt des humains

- Hôpital fonction soigner des humains

- Ecole fonction éduquer des humains

77 Ces institutions ont certes des lieux d'accueil spécialisés, mais au fond contingents l'école peut se tenir dans divers bâtiments. L'interprétation télique, pour les lieux, exige un actant humain, et indique qu'il est impliqué dans cette institution pour bénéficier de la fonction associée.

78 Il y a ici une double télicité 1) école est lexicalement défini comme ce qui est destiné à faire quelque chose au bénéfice des humains ; 2) l'actant humain de la phrase est supposé être impliqué dans l'institution pour bénéficier de cette fonction. L'interprétation de la phrase est que l'individu $\mathrm{N}$ considéré est défini comme celui qui remplit ce rôle pour l'actant humain. 
79 En somme, une institution $\mathrm{N}$ est lexicalement définie par un rôle au bénéfice des humains, et l'interprétation télique de à, traite l'actant humain localisé comme tenant de ce même rôle.

80 Il s'agit d'une généralisation empirique assez forte, impliquant que aller à l'école c'est en général en tant qu'élève, aller à l'hôpital, c'est en tant que patient, etc... Naturellement, si nous avons d'autres informations contextuelles spécifiques, un autre rôle peut être impliqué :

(41) Mon père, chirurgien renommé, allait à l'hôpital quand il fut renversé par une voiture.

81 Le mécanisme de l'anaphore associative permet d'interpréter «hôpital relatif au chirurgien » comme « hôpital dans lequel travaillait le chirurgien ». Mais même s'il en est ainsi, une phrase comme (42) admet en outre l'interprétation « hôpital dans lequel le chirurgien allait se faire soigner $»$ :

(42) - Mon père, chirurgien renommé, allait à l'hôpital quand il fut renversé par une voiture.

- Il allait au travail?

- Non. Il allait lui-même passer un scanner dans un autre hôpital.

\subsubsection{Objets-activités}

82 Les objets considérés sont des artefacts conçus afin de mener certaines activités, et l'interprétation télique associe l'actant localisé au rôle saillant dans la définition lexicale de l'entité.

(43) Le céramiste était au four. / La sculpture était au four.

83 Comme nous l'avons suggéré plus haut, l'émergence de l'interprétation télique pour les objets est moins régulière que dans le cas des institutions-lieux. En substance, les objets considérés sont des artefacts utilisés comme instruments par des humains, et les interprétations téliques se distribuent entre interprétations agentives pour les humains et interprétations en « objet affecté » pour les non-humains.

\section{Locus et telos dans d'autres tours chez le coiffeur, au coiffeur, en caisse}

On considère pour terminer, et à seule fin d'illustrer les propriétés spécifiques de la construction être (aller) à + le N, d'autres constructions du français à interprétation télique dont la relation à la localisation spéciale mériterait d'être considérée en détail.

\subsection{Chez la fleuriste, au coiffeur}

La préposition chez se combine (notamment) avec un nom désignant un humain, et localise dans le domicile de cet humain, domicile privé, ou domicile professionnel en ce qui concerne les noms de profession supposant un lieu d'exercice dédié.

La localisation d'un humain dans le lieu d'exercice d'un professionnel vient assez naturellement avec l'implicature que l'humain est en ce lieu comme bénéficiaire de l'activité professionnelle correspondante. Cela crée, en français, les couples bien connus à la boulangerie/chez le boulanger, au garage/chez le garagiste, etc. 
87 Les usages réputés fautifs du français être, aller au coiffeur ont été souvent commentés (voir en particulier Milner (1989)). Milner, comme le relève Aurnague (à paraître), insiste sur le fait suivant aller chez le coiffeur n'implique pas strictement que l'on s'y rende pour se faire coiffer, alors que aller au coiffeur l'implique strictement. Le fait est indiscutable et il est possible, à la lumière des considérations qui précèdent, de l'expliquer.

Si chez le coiffeur implique, comme on vient de l'admettre « se rendre au lieu d'exercice professionnel du coiffeur ", le fait qu'on s'y rende pour user de ses services n'est qu'une implicature. Si l'implicature est annulée, la phrase reste interprétable comme simple localisation.

89 Coiffeur, comme d'autres noms de métier, mais non tous, semble fonctionner également comme nom d'institution sociale et entrer dans la même catégorie que école, hôpital, boulangerie, etc... On en trouve confirmation dans l'usage des enseignes, et les indications d'itinéraires, par exemple :

(44) Quand tu arrives sur la place, à ta droite, tu as le coiffeur, et à ta gauche, la boulangerie.

90 Cette interprétation institution-activité est bien déterminée par une propriété lexicale du nom coiffeur, car elle s'observe pour tous les déterminants :

(45) Quand tu arrives sur la place, à ta droite, tu as un coiffeur, et à ta gauche, la boulangerie.

91 Cela conduit à définir, schématiquement, l'entrée lexicale de coiffeur de la manière suivante

Coiffeur

Individu exerçant la profession de coiffer

Institution-lieu dans lequel s'exerce cette profession

92 Cela explique, par exemple, que deux coiffeurs puisse compter des personnes, ou des entreprises exerçant le métier. La facette «institution-activité » est confirmée par des usages tels que :

(46) Le coiffeur compte plus de salariés que le boulanger.

(47) Le coiffeur a été transféré à l'autre bout du village.

93 En revanche - et c'est ce qui, selon nous, fait la différence cruciale entre les noms de profession et les noms comme boulangerie et école - les noms de profession ne sont pas d'authentiques noms de lieu. Rappelons ici que nous avons suggéré d'analyser école comme :

École

Institution-activité

Lieu dédié à l'institution

94 Le fait de traiter les noms du type école comme porteurs d'une facette lieu est destiné à expliquer qu'ils se combinent sans aucune difficulté avec tous les prédicats qui sélectionnent un lieu, alors que les noms de profession refusent clairement certains d'entre eux, notamment certaines prépositions de lieu :

(48) Dans une école /*dans un coiffeur

A l'intérieur d'une école / *à l'intérieur d'un coiffeur

Même s'il est vrai que certaines prépositions spatiales refusent moins nettement les noms de profession :

(49) L'enfant fut renversé devant le coiffeur.

(50) L'appartement est situé près du coiffeur. 

font une différence nette entre les noms de type école et les noms de type coiffeur:

(51) ? Le coiffeur a été démoli (repeint en blanc, orné d'une fresque).

Si on admet que les noms de profession ont bien une facette qui les traite comme noms d'activité-institution, mais ne sont pas lexicalement traitables comme de véritables noms de lieu, on explique, semble-t-il, tous les traits saillants qui justifient l'existence de aller au coiffeur et tous les jugements qui l'accompagnent.

\section{Pourquoi aller au coiffeur est-il légitime?}

La préposition à se construit régulièrement avec des noms d'activité, même si ce nom n'est pas un nom de lieu (aller à la gym, aller au travail). Cette construction respecte les restrictions de sélection de à et les propriétés lexicales de coiffeur qui comporte une facette polysémique "activité-institution ». Si cette construction est utilisée, elle est télique, et il n'y a pas d'autre interprétation pour la construction que «être localisé comme bénéficiaire de l'activité-institution coiffeur ».

99 Ce qui explique la remarque de Milner sur la non-ambiguité du tour, c'est que la construction avec à ne peut en aucun cas être interprétée comme une pure localisation spatiale, comme peuvent l'être des noms comme école, ou les constructions avec chez.

2. Pourquoi les puristes n'acceptent-ils pas ce tour?

Une explication d'ordre général concernant la grammaire normative peut être invoquée il y a une autre expression permettant d'exprimer la même interprétation, chez le coiffeur, et la réaction du puriste, dans un tel cas, est toujours de décréter l'une d'entre elles fautive. Il est vrai que pour certaines activités, le français dispose de trois possibilités (chez le boulanger, à la boulangerie, au boulanger).

L'argument que l'on pourrait élaborer sur la base de la remarque de Milner (l'un est ambigu, l'autre non) pour défendre la spécificité de à est de ceux dont le puriste fait en général peu de cas.

Un puriste pourrait aussi trouver que cet usage de à instaure une hétérogénéité dans ses constructions téliques à télique, dans les emplois les plus proches pour l'interprétation, se construit avec des institutions-lieux (église, école), ou des objets (piano, four) ; on perd cette unité du paradigme, si on le construit avec des noms d'agent-activité (coiffeur).

\subsection{En caisse, à la caisse}

L'étude d'ensemble des distributions de en est une question complexe, qu'il n'est pas question d'aborder ici. On se contentera de quelques remarques en lien avec la présente étude de à.

En est distingué des autres prépositions par le fait qu'en français moderne il se construit avec un nom nu, mais il a, en dehors de cela, plusieurs points d'analogie avec $\grave{a}$.

Un de ces points d'analogie avec à est que en peut se construire avec des noms d'activité ( en promenade, en chasse, en match, en guerre, en retraite), lesquels peuvent également parfois admettre à :

(52) Le seigneur était à la guerre.

(53) Le seigneur était en guerre.

(54) Pierre est en retraite.

(55) Pierre est à la retraite.

Corela, HS-12 | 2012 
Avec à, on semble garder l'idée d'une simple localisation par rapport à une occurrence de l'activité-institution, alors que en implique la caractérisation d'un type d'activité. Il est possible d'être en guerre sans être à la guerre, ce qui signale que à la guerre indique simplement la participation à une activité. En outre, et c'est sans doute dû à l'usage du défini avec $\grave{a}$, on se réfère dans ce cas à une institution sociale particulière, et non simplement au type il serait ainsi certainement plus comique d'écrire (55) que (56) :

(55) Cicéron était alors à la retraite.

(56) Cicéron était alors en retraite.

De même (57) me semble plus anthropomorphique que (58):

(57) Les fourmis étaient à la guerre.

(58) Les fourmis étaient en guerre.

Il y a également des oppositions interprétatives comme :

(59) être au concert $=$ assister au concert

être en concert $=$ donner un concert

Les prépositions à et en sont également en concurrence pour des noms d'institution-lieu, et on entend en français aussi bien (60) que (61), qui sont des quasi-synonymes :

(60) Il faudra aller en caisse se faire rembourser.

(61) Il faudra aller à la caisse pour se faire rembourser.

110 Dans beaucoup de ces cas, en s'utilise plutôt dans le langage technique, celui des professionnels, à restant d'un usage plus commun. Le point pourrait être que à, comme on l'a dit, admet deux interprétations avec les noms d'institution-lieu une interprétation de pure localisation spatiale, et une interprétation télique, alors que en n'admet pas en français moderne d'interprétation de pure localisation spatiale. Son usage serait de ce fait le seul à atteindre sans ambiguïté l'interprétation télique, d'où ses usages techniques ; être en caisse, cela ne peut pas être une simple localisation spatiale, cela signifie toujours « s'y trouver comme caissier, client, facture ou marchandise ». Il me semble par exemple, que si vous avez oublié votre propre parapluie en réglant vos achats au supermarché, vous direz plutôt (62) :

(62) J'ai oublié mon parapluie à la caisse.

111 Si en revanche il s'agit du parapluie que vous vouliez acheter, vous direz plutôt (63) :

(63) J’ai oublié mon parapluie en caisse.

112 Dans l'ensemble, ces observations contrastives supportent l'idée que de nombreuses propriétés spécifiques de à tiennent à ce que cette préposition compte le trait «lieu » parmi ses restrictions de sélection, ce qui n'est pas le cas de en $+N$.

\section{Conclusion}

113 Nous avons dans cet article donné une présentation informelle d'une hypothèse qui voit dans les tournures de type "aller à l'école» un défini fonctionnel auto-légitimé, présentation qui reprend les hypothèses principales de Corblin (2011). En outre, nous avons discuté de l'extension de cette hypothèse aux constructions de à avec des noms d'objet («être au piano ») étudiées par Aurnague (à paraître).

114 Cette hypothèse, mieux que celle d'un défini générique, et mieux que les versions non dénotationnelles (de type «incorporation» ou «idiome»), prédit les suites possibles 
effectivement licites de type ce $N$ (ou lequel?), et dérive assez naturellement l'interprétation télique qui émerge pour certaines de ces structures.

L'article a grandement bénéficié de la lecture du travail de M. Aurnague (à paraître) sur deux points essentiels. Tout d'abord, la distinction entre lieu et objet m'a conduit à discuter des interprétations téliques des objets (piano, four), alors que mon précédent travail était entièrement consacré aux noms de lieu (école, hôpital); la lecture des commentaires critiques de $\mathrm{M}$. Aurnague m'a aussi amené à préciser un point important concernant l'interprétation du défini Corblin (2011) laisse entendre que les définis téliques de ces constructions introduisent un lieu particulier dans le discours, alors qu'il est beaucoup plus juste de dire qu'ils introduisent un individu exemplaire de l'institution, la localisation de cette institution dans un lieu dédié, n'étant, comme le souligne très justement M. Aurnague, qu'une implicature.

116 Le point crucial qui me sépare de la position défendue par M. Aurnague dans son article, laquelle est, de son aveu même, par ailleurs assez proche de la mienne, est le traitement du défini comme défini associatif ou fonctionnel et comme défini spécifique, et mon argument décisif reste fondé sur la nécessité d'expliquer les successions anaphoriques et l'usage subséquent de lequel?, qui ne me semblent pas correctement expliqués dans les traitements comme ceux de Furukawa ou d'Aurnague, qui conçoivent plutôt le GN en question sur le modèle des GN génériques.

\section{BIBLIOGRAPHIE}

Aurnague, M., 2001. Entités et relations dans l'espace et son expression en basque et en français. Recherches, Université de Toulouse-Le Mirail.

Aurnague, M., 2004. Les structures de l'espace linguistique. Regards croisés sur quelques constructions spatiales du basque et du français. Leuven / Paris : Peeters.

Aurnague, M., 2009. «A cet endroit vs. dans un tel endroit : ce que à nous dit d'endroit et viceversa », Langages $173,34-53$.

Aurnague, M. (à paraître). «Quand la routine s'installe. Remarques sur les emplois de à de type 'routine sociale'«, Revue Romane.

Borillo, A., 2001. « La détermination et la préposition de lieu à en français », Lingvisticae Investigationes Supplementa 23, 85-99.

Corblin, F., 2011. « Des définis para-intensionnels : être à l'hôpital, aller à l'école », Langue française 171(3), 55-77.

Cruse, A., 1996. « La signification des noms propres de pays en anglais », in S. Rémi-Giraud \& P. Rétat (eds), Les mots de la nation. Lyon : Presses Universitaires de Lyon, 93-102.

Furukawa, N., 1986. L'article et le problème de la référence en français. Tokyo : France-Tosho.

Furukawa, N., 2010a. «L'article défini et le problème dit de l'unicité : quantité ou qualité ? ", Bulletin d'Etudes de Linguistique Française 44, 65-82. 
Furukawa, N., 2010b. « Article défini, son emploi « intensionnel » et énoncé tautologique », Bulletin d'études françaises 41, 51-71.

Hawkins, J.A., 1978. Definiteness and Indefiniteness. A Study in Reference and Grammaticality Prediction. Londres : Croom Helm.

Kleiber, G., 1999. Problèmes de sémantique. La polysémie en questions. Villeneuve-d'Ascq : Presses Universitaires du Septentrion.

Martin, R., 1986. « Les usages génériques de l'article et la pluralité », in J. David \& G. Kleiber (eds), Déterminants : syntaxe et sémantique. Paris : Klincksieck, 187-202.

Milner, J.-C., 1989. Introduction à une science du langage. Paris : Le Seuil.

Pustejovsky, J., 1995. The generative lexicon. Cambridge : M.I.T. Press.

Vandeloise, C., 1987. «La préposition à et le principe d'anticipation », Langue Française 76, 77-111.

\section{RÉSUMÉS}

Cet article considère les interprétations 'téliques' des constructions «être (aller à) à le N »; Il reprend et développe les analyses introduites dans Corblin (2011) à la lumière de commentaires récents d'Aurnague (à paraître). Il discute en particulier la possibilité d'étendre l'analyse appliquée antérieurement surtout aux noms de lieu (école, hôpital), à ce que M. Aurnague appelle des noms d'objet, et qui contrairement aux précédents n'admettent pas d'interprétation purement locative (*la mouche est au piano). L'article considère aussi la série de constructions apparentées (aller chez le coiffeur, au coiffeur, en caisse) en manifestant ce qui les oppose aux lectures téliques de à.

This paper discusses the telic reading of the constructions aller à l'école (« to go to school »), aller à la plage («to go to the beach»). It extends the analysis of Corblin (2011) in the light of recent comments by M. Aurnague (Aurnague, forthcoming). The paper discusses, in particular, the possibility of extending the analysis set up in Corblin (2011) for names of places (école, hôpital) to name of « objects » in the terminology of Aurnague, the specificity of these names being that they do not accept locative readings of the preposition $\grave{a}\left({ }^{*}\right.$ La mouche est au piano " *The fly is at the piano »). By way of comparison, the paper considers related constructions in French (aller chez le coiffeur, au coiffeur, en caisse « to go (lit.) to the hairdresser's house, (lit.) at the hairdresser, (lit.) in cash register ») and shows what distinguishes them from true telic readings of the $\grave{a}$ preposition.

\section{INDEX}

Mots-clés : défini, localisation, polysémie, facettes, routine sociale, à, chez, dans

Keywords : definite article, localization, polysemy, semantic facet, social routine 


\section{AUTEUR}

\section{FRANCIS CORBLIN}

Université Paris-Sorbonne, Institut Jean Nicod (CNRS, ENS-EHESS) \& Institut Universitaire de France 